This Section of Epidemiology and Psychiatric Sciences appears in each issue of the Journal to stress the role of the epidemiological approach to promote advances in the field of clinical psychopharmacology, with a particular attention to controversial findings. The ultimate aims are to help develop a more critical attitude towards the results of research studies published in the international literature, to promote original research projects with higher methodological standards, AND to implement the most relevant results of research in every-day clinical practice. These contributions are written in house by the journal's editorial team or commissioned by the Section Editor (no more than 1000 words, short unstructured abstract, 4 key-words, one Table or Figure AND up to ten references).

Corrado Barbui, Section Editor

\title{
Discontinuing benzodiazepines: best practices
}

\author{
G. Guaiana ${ }^{1 *}$ and C. Barbui ${ }^{2}$ \\ ${ }^{1}$ Department of Psychiatry, Western University, London, Canada \\ ${ }^{2}$ Department of Public Health and Community Medicine, Section of Psychiatry, University of Verona, Verona, Italy
}

In July 2015, the Canadian Agency for Drugs and Technologies in Health (CADTH) released a Rapid Response report summary, with a critical appraisal, on discontinuation strategies for patients with long-term benzodiazepines (BDZ) use. The CADTH document is a review of the literature. It includes studies whose intervention is BDZ discontinuation. Also, clinical guidelines, systematic reviews and meta-analyses are included. What emerges from the CADTH guidelines is that the best strategy remains gradual tapering of BDZ with little evidence for the use of adjunctive medications. The results show that simple interventions such as discontinuation letters from clinicians, self-help information and support in general, added to gradual tapering may be associated with a two- to three-fold higher chance of successful withdrawal, compared with treatment as usual. We suggest possible implications for day-to-day clinical practice.

Received 22 November 2015; Accepted 6 January 2016; First published online 28 January 2016

Key words: Benzodiazepines, discontinuation, effectiveness, guidelines.

In July 2015, the Canadian Agency for Drugs and Technologies in Health (CADTH) released a Rapid Response report summary, with a critical appraisal, on discontinuation strategies for patients with longterm benzodiazepines (BDZ) use (Canadian Agency for Drugs and Technology in Health, 2015). BDZ use is widespread. BDZ are very frequently prescribed (Baldwin et al. 2012; Huerta et al. 2015) and have been used in anxiety disorders (Berney et al. 2008; Offidani et al. 2013), although its use declined to the advantage of antidepressants (AD; Berney et al. 2008; Katzman et al. 2014).

BDZ use is controversial. On the one hand they tend to be quite effective, with a short onset of action and

\footnotetext{
* Address for correspondence: Dr G. Guaiana, Department of Psychiatry, St. Thomas Elgin General Hospital, 189 Elm Street, St. Thomas, Ontario N5R 5C4, Canada.

(Email: gguaiana@uwo.ca)
}

good tolerability profile (Stahl, 2002). However, there have been increasing concerns over their use, as they can be addictive in the long-term (Lader, 2011) and can cause sedation and drowsiness (Lader, 2011). Also, its use may be problematic in seniors as they can cause increased risk of falls, cognitive decline and delirium (Marra et al. 2015). As a consequence, there has been some debate on whether BDZ are dangerous medications, to be used as little as possible (Ashton, 2005), or whether its use has been prematurely discounted (Offidani et al. 2013).

In any case, BDZ use can be problematic and can lead to adverse effects. So, long-term use should be carefully evaluated and, if necessary, BDZ dose should be reduced. According to the current evidence, BDZ should be used for a short time (Baldwin et al. 2014), or else it may be associated with tolerance or lack of efficacy. This is why a document like the one released by the $\mathrm{CADTH}$, on how to discontinue 
BDZ, can be helpful, as it can offer some guidance on how to safely and effectively stop a potentially problematic medication.

The CADTH document is a review of the literature. It includes studies whose intervention is BDZ discontinuation. Also, clinical guidelines, systematic reviews and meta-analyses are included. The document excludes studies published prior to 2010 and guidelines outside Australia, Canada, the UK and the USA. The population examined is composed by adults in a community setting who took BDZ for longer than 3 months. The aim of this review is to examine the clinical evidence on the BDZ discontinuation strategies. Moreover, another goal is to explore the evidencebased guidelines regarding discontinuation of longterm (i.e. longer than 3 months) BDZ use.

After a comprehensive literature search, the document included three systematic reviews, five randomized controlled trials (RCTs) and three non-RCTs. Mean age for the population ranges from 41 to 79 years. The most common intervention in the included studies is gradual BDZ dose tapering combined with some kind of psychotherapeutic, psycho-educational or psychopharmacological intervention. The comparator is a treatment as usual protocol or a slow tapering without any intervention. The authors of the document did not find any guidelines for BDZ tapering in the literature. The results show that simple interventions such as discontinuation letters from clinicians, selfhelp information and support in general, are associated with a better chance of successful withdrawal, compared with treatment as usual. The systematic review found that patients who receive simple interventions are twice as likely to completely withdraw from BDZ, compared with controls. If cognitivebehavioural therapy is included as intervention in addition to gradual dose tapering, the percentage of people discontinuing BDZ is between 65 and $85 \%$, compared with dose tapering alone, where the percentage is only $25-54 \%$. Supervised BDZ withdrawal was associated with a higher chance of success. Education interventions were also associated with a higher percentage of discontinuation, $45 \%$, compared with usual care, where only $15 \%$ of subjects were able to successfully withdraw from BDZ. With regard to psychopharmacological interventions, it appears that adjuvant medications are not associated with better outcomes. In particular, some of the studies included showed that melatonin was no better than placebo in facilitating BDZ discontinuation (38.1 $v$. $47.7 \%$ respectively). Another medication that was included in the comparison was pregabalin, which was associated with successful BDZ withdrawal, with $52 \%$ of the subjects achieving BDZ-free status after 12 weeks. However, the study examining this issue was uncontrolled. Also, hydroxyzine and valerian have been studied. Data show that gradual reduction of BDZ use, in association with hydroxyzine and valerian is associated with abstinence from BDZ in $80.4 \%$ of the cases at 6-month follow-up.

What emerges from the CADTH guidelines is that the best strategy remains gradual tapering of BDZ with little evidence for the use of adjunctive medication. Simple interventions may increase the chance of successful withdrawal by two to three times and they may be valuable aids in assisting with BDZ discontinuation.

However, there are still no clear specified guidelines on successful discontinuation. For this reason, the CADTH document is a welcome addition to the literature on $\mathrm{BDZ}$ as it gives some guidance on the best strategy to discontinue BDZ. It must be said, thought that the quality of the studies included is not very high and further research is needed. A topic that may also be interesting is what additional psychosocial intervention is most effective in BDZ discontinuation. The studies included in the CADTH review, compared BDZ interventions with treatment as usual. Comparing different types of additional psychosocial interventions may offer some help and can be potentially useful in developing suitable guidelines in BDZ discontinuation. What clinicians should in any case consider, though, is the fact that it is possible to successfully discontinue $\mathrm{BDZ}$, if it is needed.

\section{Acknowledgements}

None.

\section{Financial Support}

No financial support was received for this paper from any funding agency, commercial or not-for-profit sectors.

\section{Conflict of Interest}

None.

\section{References}

Ashton H (2005). The diagnosis and management of benzodiazepine dependence. Current Opinion in Psychiatry 18, 249-255.

Baldwin DS, Allgulander C, Bandelow B, Ferre F, Pallanti S (2012). An international survey of reported prescribing practice in the treatment of patients with generalised anxiety disorder. World Journal of Biological Psychiatry 13, 510-516. 
Baldwin DS, Anderson IM, Nutt DJ, Allgulander C, Bandelow B, den Boer JA, Christmas DM, Davies S, Fineberg N, Lidbetter N, Malizia A, McCrone P, Nabarro D, O'Neill C, Scott J, van der Wee N, Wittchen HU (2014). Evidence-based pharmacological treatment of anxiety disorders, post-traumatic stress disorder and obsessive-compulsive disorder: a revision of the 2005 guidelines from the British Association for Psychopharmacology. Journal of Psychopharmacology 28, 403-439.

Berney P, Halperin D, Tango R, Daeniker-Dayer I, Schulz P (2008). A major change of prescribing pattern in absence of adequate evidence: benzodiazepines versus newer antidepressants in anxiety disorders. Psychopharmacology Bulletin 41, 39-47.

Canadian Agency for Drugs and Technology in Health (2015). Discontinuation strategies for patients with long-term benzodiazepine use: a review of clinical evidence and guidelines. Retrieved 20 October 2015 from https://www. cadth.ca/sites/default/files/rc0682-bzd_discontinuation_ strategies_final_0.pdf.

Huerta C, Abbing-Karahagopian V, Requena G, Oliva B, Alvarez Y, Gardarsdottir H, Miret M, Schneider C, Gil M, Souverein PC, De Bruin ML, Slattery J, De Groot MCH, Hesse U, Rottenkolber M, Schmiedl S, Montero D, Bate A, Ruigomez A, García-Rodríguez LA, Johansson S, de Vries F, Schlienger RG, Reynolds RF, Klungel OH, de Abajo FJ (2015). Exposure to benzodiazepines (anxiolytics, hypnotics and related drugs) in seven European electronic healthcare databases: a cross-national descriptive study from the PROTECT-EU Project. Pharmacoepidemiology and Drug Safety. doi: 10.1002/pds.3825.

Katzman MA, Bleau P, Blier P, Chokka P, Kjernisted K, Van Ameringen M; Canadian Anxiety Guidelines Initiative Group on behalf of the Anxiety Disorders Association of Canada/Association Canadienne des troubles anxieux and McGill University, Antony MM, Bouchard S, Brunet A, Flament M, Grigoriadis S, Mendlowitz S, O'Connor K, Rabheru K, Richter PM, Robichaud M, Walker JR (2014). Canadian clinical practice guidelines for the management of anxiety, posttraumatic stress and obsessive-compulsive disorders. BMC Psychiatry 14 (Suppl. 1), S1. doi: 10.1186/ 1471-244X-14-S1-S1.

Lader M (2011). Benzodiazepines revisited - will we ever learn? Addiction 106, 2086-2109.

Marra EM, Mazer-Amirshahi M, Brooks G, van den Anker J, May L, Pines JM (2015). Benzodiazepine prescribing in older adults in U.S. Ambulatory Clinics and Emergency Departments (2001-10). Journal of the American Geriatric Society 63, 2074-2081.

Offidani E, Guidi J, Tomba E, Fava GA (2013). Efficacy and tolerability of benzodiazepines versus antidepressants in anxiety disorders: a systematic review and meta-analysis. Psychotherapy and Psychosomatics 82, 355-362.

Stahl S (2002). Don't ask, don't tell, but benzodiazepines are still the leading treatments for anxiety disorder. Journal of Clinical Psychiatry 63, 756-757. 